\title{
An Experimental Investigation of Closed-Loop Impedance Pumping in a Compliant, Elastic Tube Millistructure by Variation of Perturbation Location
}

\author{
Nicholas G. Garafolo*, Bryan C. Rich, Matthew J. Cymbal \\ Department of Mechanical Engineering, The University of Akron, Akron, OH 44325, U.S.A \\ *Corresponding author: nicholas.g.garafolo@uakron.edu
}

\begin{abstract}
Flow through a fluid-filled compliant tube microstructure subject to periodic perturbations is not widely reported. An understanding of this phenomena is sought herein, as it may be useful for design of biological flows in microfluidic devices. An experiment conducted at the milli-scale to study the fluid flow produced within a closedloop network of tubing, having a compliant millitube section subject to periodic perturbations from a probe is presented, and provides a basis for a micro-scale experiment. To test the fluid response, the boundary of the compliant millitube section was periodically perturbed by the probe at a variety of frequencies at five locations. Experimental results demonstrate fluid circulation within the closed-loop of tubing, which varies significantly with the frequency and location of applied perturbations. Overall, the study herein illustrates a unique design for pumping which utilizes periodic vibrations of a compliant tube structure to create a net positive displacement of fluid.
\end{abstract}

Keywords: impedance pumping, fluid-structure interaction, elastic tube

Cite This Article: Nicholas G. Garafolo, Bryan C. Rich, and Matthew J. Cymbal, "An Experimental Investigation of Closed-Loop Impedance Pumping in a Compliant, Elastic Tube Millistructure by Variation of Perturbation Location." Journal of Mechanical Design and Vibration, vol. 5, no. 1 (2017): 1-10. doi: $10.12691 / j m d v-5-1-1$.

\section{Introduction}

Fluid flow within a compliant tube microstructure, subject to periodic perturbations, is not widely reported. A microfluidic flow under these conditions is similar to vascular flow; flow through vessels which are compliant and, at times, are subject to perturbations from body movement or contact with external stimuli. Moser et al. have suggested, based on the works of Weber, Donders, Ozanam, and Liebau, that boundary movement of vessels can produce a pumping effect which assists in the circulation of blood that the heart cannot sustain alone [1], and it was even suggested to be an optimal use of energy [2]. Future devices will likely be engineered to mimic such biological microflows and so a method for recreating this valveless pumping by perturbation of compliant tube microstructures will be needed. Therefore, it was of interest to quantify how a fluid, inside of a compliant tube microstructure, responds to periodic boundary movement and how that might produce pumping.

Many microfluidic pumps in use today use a controlled boundary movement to move a fluid. Pumps which operate based on this principle are classified as displacement pumps. The challenge with displacement pumps is to control the flow direction and a number of different methods, employing various check valve structures have been developed that achieve such flows $[3,4,5,6,7]$. A special method, called acoustic streaming, does not use check valves but instead propagates a traveling wave at the boundary to produce unidirectional pumping. A common mechanism to almost all these displacement micro-pumps is the deformation of a thin linear elastic boundary to cause fluid flow where the deformation is directly proportional to the size of the actuator used. More representative of a real biological flow, however, is the use of a fully compliant tube structure that would more easily deform under a load and propagate a traveling wave. Therefore, it was expected to require less actuation at lower frequencies in order to produce an effect similar to traditional micro pumps. The material costs, power consumption, and weight of a pump can be reduced if less actuation is needed.

It was hypothesized that development of a novel, affordable, and effective micro-pump is possible using controlled perturbations of a compliant tube structure. Accordingly, the research objective of this study was to quantify the performance of a pump utilizing the vibrations of the elastic structure as the carrier method.

\subsection{Valveless, Impedance Pump}

Perturbations of a compliant structure will produce a traveling wave at the boundary called a Rayleigh wave. This wave propagates equally in all directions unless it encounters destructive or constructive interference. A unidirectional wave is produced which can facilitate unidirectional pumping if the wave is destructed in all but one direction. This is one example of the acoustic 
streaming methods used to produce pumping of fluid [8]. One way to diminish a Rayleigh wave is at the boundary of two solids with different impedances. At this location the wave can be both damped and reflected. Perturbations of a compliant structure near a solid-solid boundary produce waves which are either destructed or reflected and added to the propagated wave in the opposite direction, creating a mainly unidirectional wave that can facilitate pumping. This is called impedance pumping as first noted by Liebau in 1954 [9] which has been applied to microfluidic applications [10,11,12].

A valveless, impedance pump consists of a compliant section of fluid channel coupled to more rigid fluid channels that have a higher impedance. The pumping is dependent on wave reflection and interference and so the compliant material allows for wave propagation while the rigid material allows for wave reflection, Figure 1. Periodic compressions of the compliant section at an asymmetric location creates an imbalance of wave reflections which accumulate and drive flow. An advantage of this pump is that it is valveless, reversible, and requires few components.

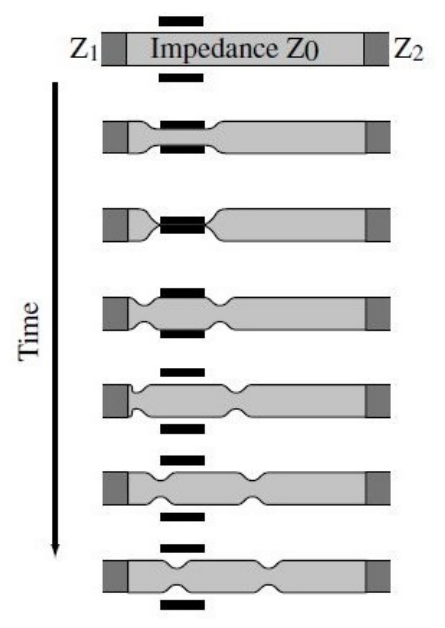

Figure 1. Pinching scheme and wave reflections of an impedance pump [13]

An impedance pump operating similar to the proposed pumping principle was developed and tested by Ottesen [14]. The apparatus consisted of a closed ring of tubing made of two different rubbers and filled with an incompressible fluid. It was noted that periodically compressing the tube at an asymmetric location causes the internal fluid to flow and the flow rate depends on the frequency of compressions, elasticity of the tubes, compression ratio, and type of compression. The experiment was conducted at the macro-scale, however, and may not hold true for a micro-scale flow, as many fluid flow principles have been noted to differ at this scale.

A micro impedance pump was developed by Wen et al. [12] in which a thin-walled section of channel, made of nickel, was actuated by a single PZT element placed at an asymmetric location to induce pumping. Results of the experiment demonstrated a water level difference in two vertical capillaries which indicates an accumulation of a pressure gradient and therefore the potential to drive flow. Furthermore, peak pumping was reported at a frequency as low as $25 \mathrm{~Hz}$. The operating principles of this pump are very similar to the proposed pumping method to be studied except for the use of a compliant material and closed-loop network of tubing.

Lee et al. [10] created a similar impedance pump using a single compliant PDMS membrane driven by electromagnetic actuation. Results of an experiment demonstrated a water level difference in two vertical capillaries which indicates the potential to drive flow. Flow was only evaluated at frequencies between $100 \mathrm{~Hz}$ and $500 \mathrm{~Hz}$ where peak pumping occurring at $200 \mathrm{~Hz}$. Deformation of a single compliant membrane may not provide the same pumping effect as deformation of a compliant structure.

Several efforts have been made in the area of impedance pumping since its discovery by Liebau. The majority of these works, however, describe the pinching of a tube at the macro scale or the compression of a compliant membrane at the micro scale to produce a pumping effect

\subsection{Peristaltic Pump}

Pertubations of a fluid-filled compliant tube structure will cause the fluid and structure to deform. If the fluid is nearly incompressible it will undergo an undirected displacement per the method of displacement pumping. When the compliant tube structure is pinched closed, subsequent perturbations will produce a unidirectional displacement of fluid. This method is known as peristaltic pumping and has been applied to microfluidic applications.

A peristaltic pump consists of a single loop of tubing with a compliant section that can be squeezed externally by three or more rollers or actuators to produce pumping. Each roller or actuator acts to displace volume for pumping and as a valve to rectify flow. By compressing the tube with rollers or actuators in a particular sequence a net positive displacement of fluid was achieved [3].

Koch et al. [15] developed a miniature rotary peristaltic pump that pumps fluid by deforming a micro channel integrated into a compliant microchip. The miniature rotary pump was also tested with traditionally used compliant tubing to provide a comparison of pumping performance. It was determined that both pumps had similar performance and the first was of benefit to interface with microfluidic devices.

Shkolnikov et al. [16] created a miniature peristaltic pump controlled by a single actuator. The design of a special structure was presented which surrounds a compliant tube and compresses it in such a way that recreates the effect of a traditional three-actuator peristaltic pump. Their pump is also simpler to control than other three-actuator pumps which require careful timing of the actuation sequence to produce pumping.

Graf and Bowser [17] developed a peristaltic micropump which uses actuation of cantilever beams to compress a compliant fluid channel and produce pumping. During operation the beams compress the compliant channel to completely close off flow. A sequential actuation of three of these beams produces a net positive displacement of fluid similar to a rotary peristaltic pump.

Several methods for pumping a fluid peristaltically though a compliant channel have been developed. Similar to all these pumps are the use of a three-part pumping mechanism that compresses compliant tubing to produce a 
net positive displacement of fluid. This compression of compliant tubing is similar to the pinching method of impedance pumping, but peristaltic pumping is different in that two additional actuators or rollers are employed to control flow direction instead of allowing impedance differences in the system to determine flow direction.

Fluid displacement by perturbation of a compliant tube microstructure appears to be a variation of the presented compliant pumps and may produce a different pumping effect. Presented herein, is an experimental study of this phenomena at the milli-scale by applying a variety of pertubations to a compliant millitube section of a closedloop system of tubing filled with fluid. Results of this experiment provide a quantification of the pumping effect and basis for a micro-scale experiment.

\section{Theory of Vibrations and Fluids}

Periodic boundary perturbation of a compliant tube structure was hypothesized to operate similar to an impedance pump. The pumping effect was, therefore, expected to be dependent on the imbalance of traveling waves and the fluid displacement caused by a coupled boundary displacement. This suggests that the theory of structural vibrations and fluids are useful for understanding and quantifying the pumping behavior. A review of structural vibrations and fluids is presented as it relates to the study at hand.

\subsection{Fluids}

Boundary deformation of the compliant test section will cause a coupled displacement of fluid within the closedloop system. For incompressible fluids in particular, the volume of the fluid displaced through the system for a single downstroke is equal to the volume of the fluid displaced by the boundary based on continuity equation:

$$
\nabla \cdot u_{\text {fluid }}=0
$$

where $\boldsymbol{u}_{\text {fluid }}$ (bold) is the velocity vector of the fluid. During an upstroke, the compliant tube section is free to return to its original configuration and will undergo a reverse displacement to do so causing a coupled reverse displacement of fluid volume. This suggests that equal volumes of fluid will be displaced over a downstroke and upstroke causing it oscillate rather than produce a net pumping effect.

Periodic perturbations of the compliant tube supply it with kinetic energy. Some of the energy is converted into potential energy as the compliant tube deforms, and some of the energy is converted into vibrational energy causing the compliant tube to vibrate. There is momentum associated with the motion of the tube due to periodic deformation and vibrations, some of which is transferred into the fluid at the boundary and causes fluid to flow according to the Navier-Stokes equations:

$$
\begin{aligned}
& \rho \frac{\partial u_{\text {fluid }}}{\partial t}+\rho\left(u_{\text {fluid }} \cdot \nabla\right) u_{\text {fluid }} \\
& =\nabla \cdot\left[-p I+\mu\left(\nabla u_{\text {fluid }}+\left(u_{\text {fluid }}\right)^{T}\right)\right]+F
\end{aligned}
$$

where $\rho$ is the density of the fluid, $\boldsymbol{F}$ (bold) is the body force vector, $p$ is the pressure, $\boldsymbol{I}$ (bold) is the identity matrix, and $\mu$ is the dynamic viscosity of the fluid. Momentum supplied by perturbations which are applied perpendicular to the boundary of the compliant tube may not produce a positive net fluid displacement as flow through tubes is usually caused by a momentum component parallel to the boundary. This suggests that any net positive fluid displacement occurring must be due to momentum transferred by vibrations which create a traveling wave at the boundary and have a velocity component parallel to the boundary.

\subsection{Vibrations of Elastic Structures}

The success of this research is largely reliant on the vibration theory of mechanical structures as fluid flow is expected to be induced by accumulations of a traveling waves caused by periodic perturbation of the compliant tube section. Wave accumulations in a system are known to occur at its resonance frequency and so the amplitude of wave accumulations, and therefore pumping, should be maximum at the natural frequency of the compliant test section. To determine the natural frequency, it is necessary to first identify how the structure is supported. The compliant tube section will be securely supported at both ends by the test apparatus. It is assumed that these conditions are equivalent to the classical definition of a fixed-fixed support. This classical support defines a general equation for the natural frequency of mechanical structures which applies a set of non-dimensional frequencies depending on the support conditions. This general equation is as follows:

$$
\tilde{\omega}_{k}=\omega_{k} \sqrt{\frac{E I}{\rho A L^{4}}}
$$

where $\widetilde{\omega}_{k}$ is the natural frequency and is equal to the nondimensionalized natural frequency multiplied by the square root of several material properties (e.g., density, area, length, Young's modulus, area moment of inertia). Each non-dimensionalized frequency corresponds to a different vibration mode shape starting with a single wave with one crest and increasing by one crest for each consecutive non-dimensional frequency. For this application a minimum of two crests is desired which corresponds to the non-dimensional frequency of a fixedfixed structure, $\omega_{2}$

It is also possible to determine the non-dimensional natural frequencies by solving the roots of a characteristic equation. As with the non-dimensional frequencies, these equations vary based on the support conditions. In this case of a fixed-fixed structure, the characteristic equation of the vibration modes is:

$$
\cos \lambda^{1 / 4} \cosh \lambda^{1 / 4}=1
$$

where the roots, $\$$ lambda $\$$, of the characteristic equation are related to the non-dimensional natural frequency by:

$$
\omega_{k}=\sqrt{\lambda_{k}}
$$

Theoretically, there are an infinite number of solutions to this equation, meaning that for any given structure there 
are an infinite number of natural frequencies and resulting mode shapes at which an object will vibrate. While the instrumentation may not exist to utilize them all, there are still many frequencies in the operational range which can be applied to excite the complaint test section [18]. Furthermore, the energy output of excitation instruments is limited and so the displacement amplitude of an excitation must be decreased to allow for an increase in frequency. In general, as the excitation frequency is increased the displacement amplitude is reduced to essentially nothing so that no boundary displacement and coupled fluid displacement will occur. This is also somewhat true in the reverse direction in that no displacement occurs for an excitation frequency of $0 \mathrm{~Hz}$. Therefore, an excitation at the lowest natural frequency of the compliant test section should produce the largest fluid displacement as it would result in the largest boundary displacement and amplitude of traveling waves.

\section{Experimental Evaluation of Pumping}

In order to quantify the circulation pumping effect caused within a closed-loop system an experiment was setup to facilitate a controlled application of perturbations to the compliant test section. The perturbations were varied by location and frequency in a series of tests and the resulting pumping effects were quantified.

\subsection{Compliant Test Section}

A commercially available thin-walled latex rubber tube, 0.2 inch in diameter, was selected for testing, Figure 3. The tube was considered very compliant because of its low elastic modulus and thin-wall which allow it to easily deform under a small load. This was advantageous because a small load was sufficient to provide a large and easily measureable coupled fluid-structure response. The test specimen was created by cutting section from the tubing 2-3 inches in length. Each end of the test specimen was stretched over a larger diameter glass tube on the test apparatus to create a water tight seal. The gap between the glass tubing that the test specimen spanned was 1.25 inches making this its effective length.

\subsection{Test Apparatus}

A test apparatus was constructed to facilitate the quantification of fluid pumping when the compliant millitube structure was perturbed. It consisted of several glass fixtures which were joined with an O-ring seal to create an incomplete ring of glass tubing to be completed by a complaint test section, Figure 2. Two semicircular glass tubing fixtures with an effective bending radius of 1.75 inches were used to shape the tubing loop and are $11 \mathrm{~mm}$ in diameter with a $2 \mathrm{~mm}$ thick wall. Two ends of the curved glass fixtures were connected by a flowmeter spanning 12 inches. Specific features of this flowmeter include a full scale flow rate of $\pm 2000 \frac{\mu L}{s}$ that is linearly mapped to a $0-10$ $\mathrm{V}$ output, an operating temperature range of $5-50^{\circ}$, and an accuracy of the larger between of $5 \%$ of the measurement taken and $0.125 \%$ of the full scale flow rate. The remaining ends each supported a smaller straight glass tube $6 \mathrm{~mm}$ in diameter and 6 inches in length with a $1 \mathrm{~mm}$ thick wall and are replaceable adapters for interfacing test apparatus with different compliant test sections. When fully assembled the incomplete glass loop had a gap of 1.25 inches where the compliant millitube structure was attached. Also integrated into the test apparatus were two stopcocks to regulate the internal pressure and threaded ports to interface sensors for measuring variables such as temperature and pressure. Constructing the test apparatus in this way allowed for versatility of the measurement devices and test sections used in experiments.

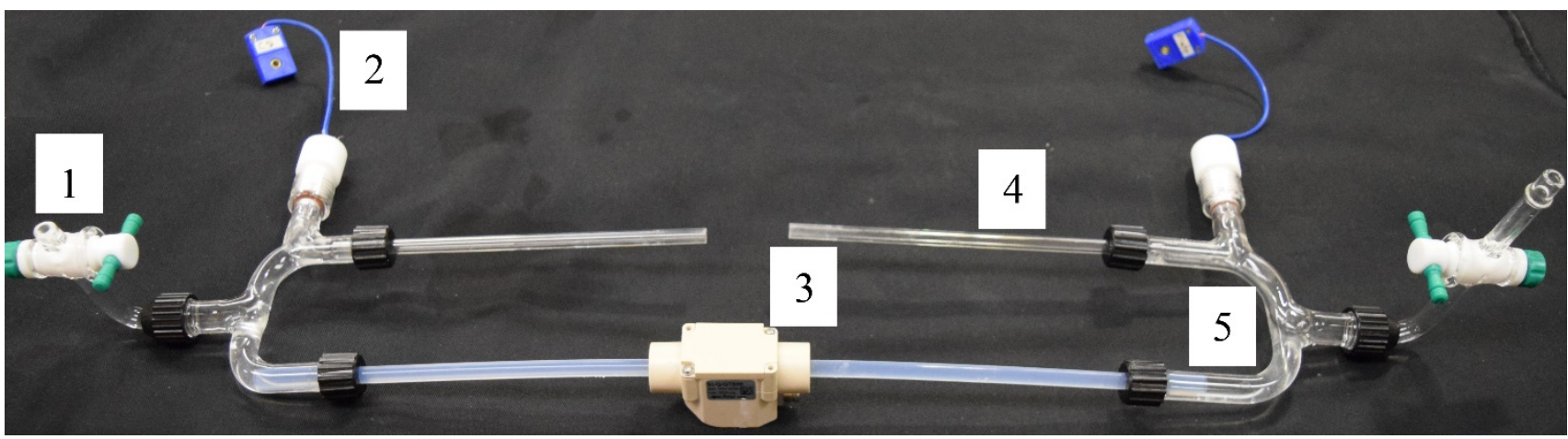

Figure 2. Image of the assembled experimental apparatus comprising of (1) stopcocks for pressure regulation, (2) threaded ports with thermocouples, (3) a flow sensor, (4) glass tubing for interfacing the test section, (5) curved glass tubing to form the flow loop

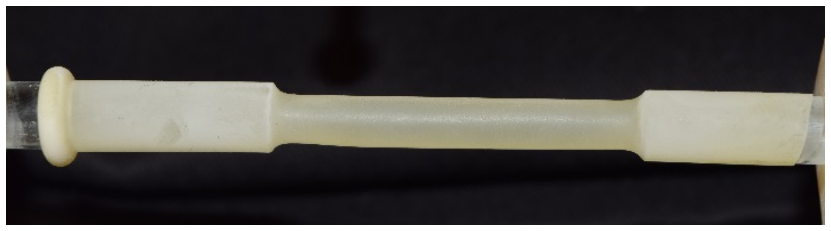

Figure 3. Image of compliant test section

\subsection{Experimental Approach}

Testing of the fluid response caused by periodic perturbation of the compliant test section was carried out in parametric sweeps. A ball-point probe attached to a dynamic shaker was aligned to one of several locations along the test section and then compressed into the test section 0.14 inch to ensure consistent contact was made. 
Perturbations were applied at a gain of $3.1 \mathrm{~V}$, and at several locations along the length of the test specimen of 0.125 inch, 0.375 inch, 0.625 inch, 0.875 inch, and 1.125 inches and correspond to positions on the test section as shown in the following diagram, Figure 4. A sinusoidal function with an initial amplitude of $1 \mathrm{~V}$ peak to peak was defined and its amplitude was varied with an amplifier to $3.1 \mathrm{~V}$ and is expressed by the following equation:

$$
d(t)=d_{f} \sin (2 \pi f t)
$$

where $t$ is time, $f$ is the frequency, $d_{f}$ is the maximum displacement amplitude at a given frequency, and $d(t)$ is the amplitude at a given time. The selected gain corresponds to a peak-to-peak displacement amplitude that varies with frequency, Figure 5. The frequency of the signal was varied from $10-50 \mathrm{~Hz}$ and the flowrate of fluid inside the apparatus was measured with a flowmeter. No significant flow response was detected at frequencies outside this range. Through these series of experiments the pumping performance of the compliant test section was obtained for perturbations applied at a variety of locations and frequencies.

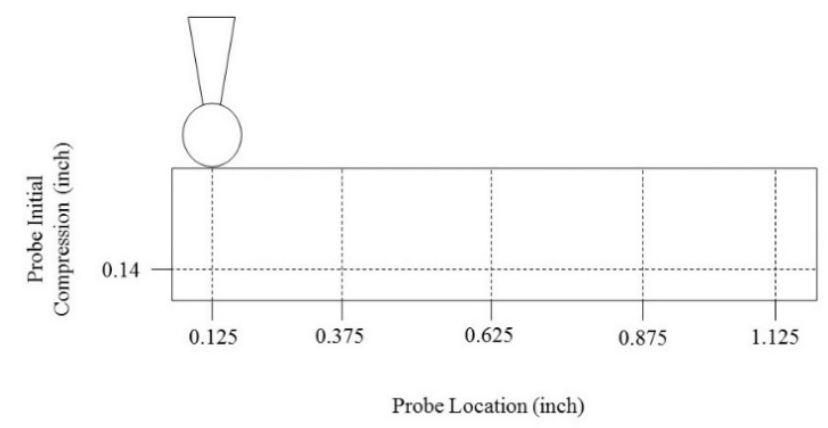

Figure 4. Diagram of test specimen showing a mapping of the probe locations tested

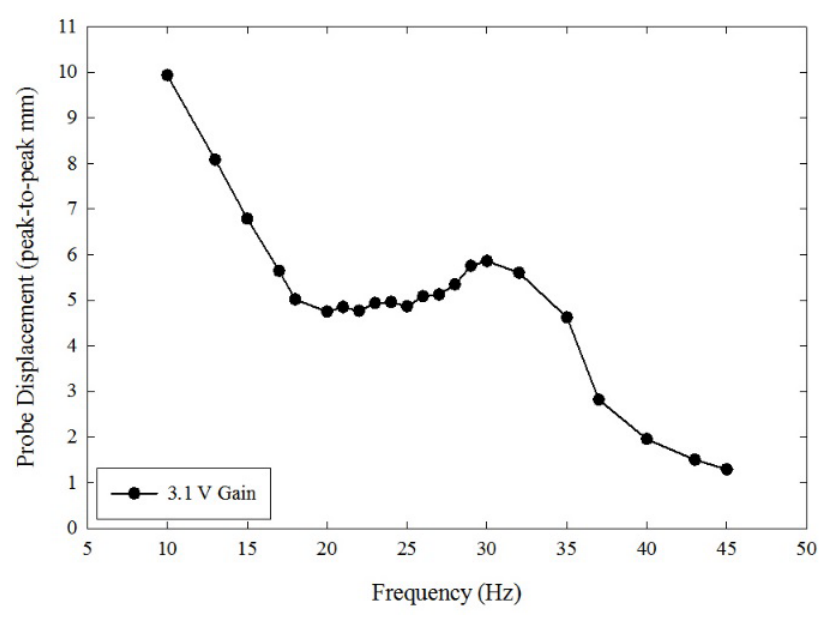

Figure 5. Graph showing the peak-to-peak displacement of the probe at the selected gain for the band of frequencies studied

\subsection{Bias}

Quantification of the fluid response was dependent on the conservation of fluid flow from the point of perturbation to the flow sensor and the capability of the flow sensor to measure it accurately. According to the manufacturer, sensor measurements are accurate to within the larger of $5 \%$ of the measured flowrate and $0.125 \%$ of the sensor's full scale flowrate of $\pm 2000 \frac{\mu L}{s}$. For most of the flowrates measured, which are below $50 \frac{\mu L}{s}$, the larger error was the one based on the full scale flowrate which simplifies to $\pm 2.5 \frac{\mu L}{s}$. This error was quite significant as it was equal to or larger than many of the flowrates measured.

\subsection{Precision}

To determine the precision error associated with repeatability of experimental conditions, ten frequency sweeps were conducted at an arbitrary location. From these measurements, the mean, standard deviation, and precision error were calculated from the following equations:

$$
\begin{gathered}
\bar{x}=\frac{1}{N} \sum_{1}^{N} x_{i} \\
\sigma=\sqrt{\frac{1}{N} \sum_{1}^{N}\left(x_{i}-\bar{x}\right)^{2}} \\
\phi=t_{v, P} \sigma .
\end{gathered}
$$

where $\bar{x}$ is the arithmetic mean, $N$ is the number of tests, $\sigma$ is the standard deviation, $x_{i}$ are the values of the flow measurements, $\phi$ is the precision error, and $t_{v, P}$ is the $\mathrm{t}_{\text {statistic }}$ of $v+1$ data samples with a $\mathrm{P}$ confidence level as determined using a Student's t-distribution.

The standard deviation of experimental measurements was added to the error in flowmeter measurements to determine the total uncertainty and is presented along with the mean, Figure 6. From this study it was concluded that the flowrate measurements were accurate to within about $\pm 2.5-4 \frac{\mu L}{s}$, depending on the frequency being applied.

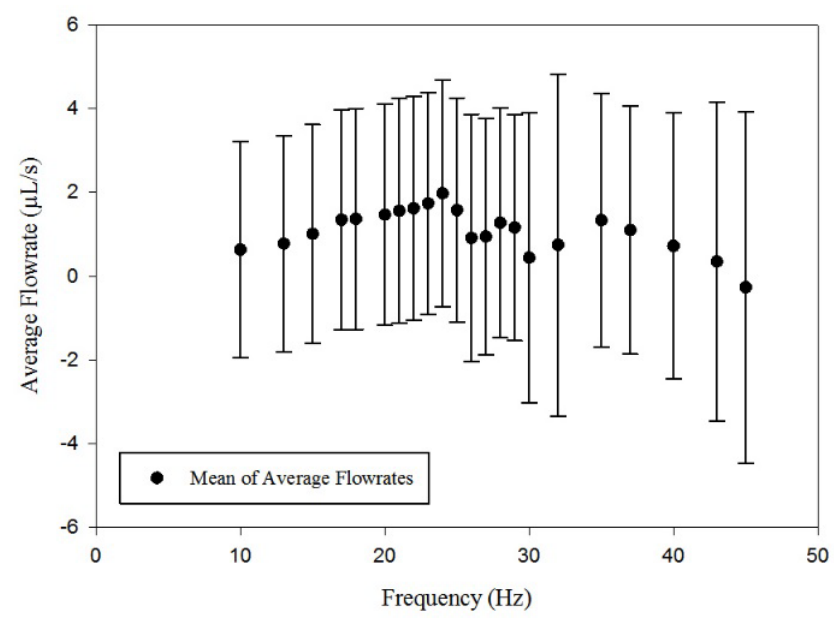

Figure 6. Graph showing an average of ten measured flow responses and their total uncertainty in resolution and repeatability

\section{Results and Discussion}

Flowrate measurements taken during the experiment revealed a sinusoidal oscillation of internal fluid whose 
frequency was synchronous with the applied perturbation, Figure 7. The oscillatory fluid response exhibited both large and small peaks in some cases, as the one shown, and were assumed to be the result of wave reflections occurring at the interface of the test section and test apparatus, as described by impedance pumping. At some of the frequencies studied the oscillatory fluid response was offset from zero which indicates pumping. The wave shape and amplitude of the fluid oscillations also varied with frequency. For each flowrate measurement the offset of the oscillatory fluid response was determined by taking the average of the flow response and was defined to be the effective pumping rate. Changes in this pumping rate were studied at a small band of frequencies over several locations.
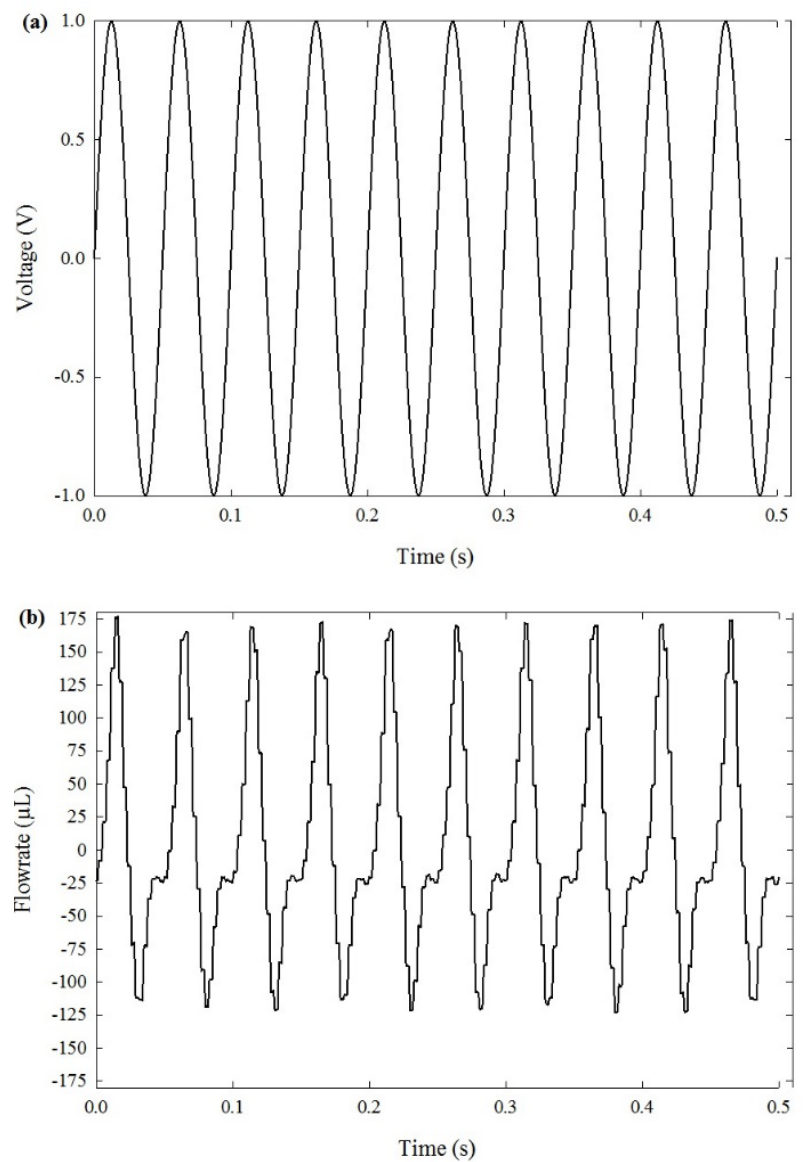

Figure 7. Graphs showing the (a) waveform generator excitation signal which was synchronous with the (a) oscillatory fluid response

\subsection{The Effect of Location on Pumping}

Variations in the average flow response resulting from perturbations applied at the five locations along the test section are presented. At each location the average flow response over a sweep of frequencies is presented.

Results illustrated the average flow response caused by applying perturbations to the test section at a locations of 0.125 inch measured from an end, Figure 8. A significant variation in magnitude and direction of the average flow response over the range of frequencies is observed with a very small positive peak at $21 \mathrm{~Hz}$ and several larger negative peaks, the largest of which is at $27 \mathrm{~Hz}$. The large peak was observed to occur due to complete pinching closed of the compliant test section over a perturbation cycle which appears to cause the vibrational effects inducing a flow response to be greatly amplified. In general, the average flow response has a magnitude of $0 \frac{\mu L}{s}$ except at the band frequencies $20-35 \mathrm{~Hz}$ where it significantly increases in magnitude.

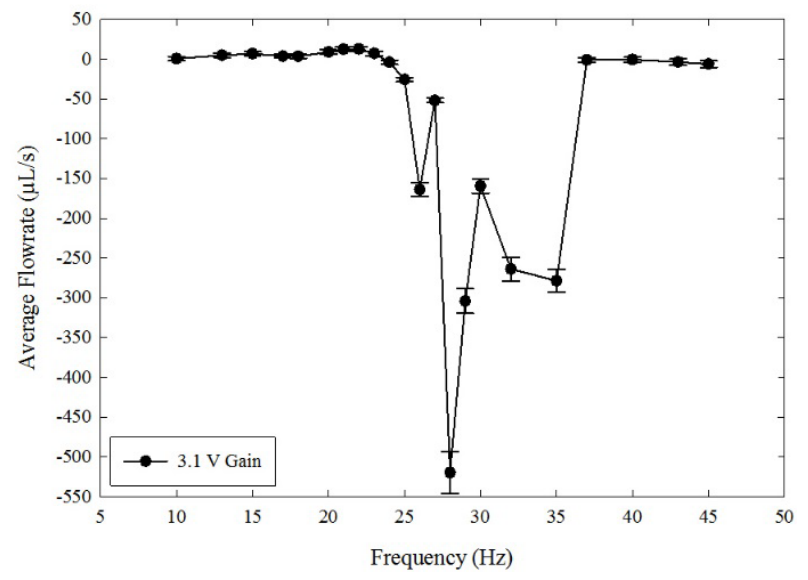

Figure 8. Graph showing the average flow response at several frequencies for a $3.1 \mathrm{~V}$ gain excitation applied at a location 0.125 inch from the end.

The average flow response caused by applying perturbations to the test section at a location of 0.375 inch, measured from an end are illustrated in Figure 9. A much smaller variation in magnitude of the average flow response over the range of frequencies is observed with a negative peak at $35 \mathrm{~Hz}$. In general, the average flow response has a magnitude of $0-1 \frac{\mu L}{s}$ except at the band of frequencies $23-40 \mathrm{~Hz}$ where it increases in magnitude. There is no obvious reason as to why the average flow response did not have a magnitude of $0 \frac{\mu L}{s}$ and so it was assumed that this result was an error which reflected an average flow response of $0 \frac{\mu L}{s}$ as supported by the uncertainty analysis. A decay in magnitude of the average flow response after the peak frequency is evident and occurs very gradually compared to the growth up to peak, but this was assumed to be due to measurement error based on the uncertainty analysis as this non-symmetry was not observed at some other locations.

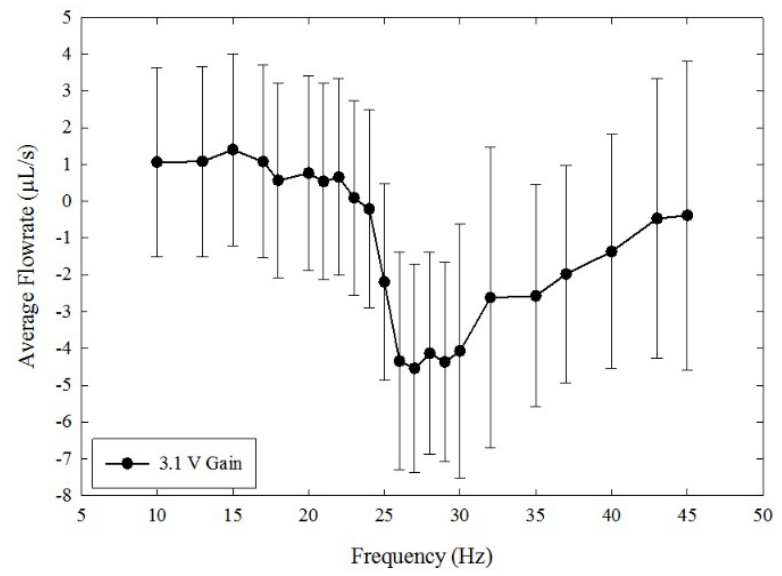

Figure 9. Graph showing the average flow response at several frequencies for a $3.1 \mathrm{~V}$ gain excitation applied at a location 0.375 inch from the end 
Results illustrating the average flow response caused by applying perturbations to the test section at a location of 0.625 inch, measured from an end, are shown in Figure 10. This location corresponds to the middle of the test section. Little variation in magnitude of the average flow response over the range of frequencies is observed with a negative peak at $35 \mathrm{~Hz}$ that cannot be verified based on the uncertainty analysis and suggests that the average flow response is entirely zero for any frequency applied at this location. In general, the average flow response has a magnitude of $-1-0.5 \frac{\mu L}{s}$ - except at the band of frequencies $30-37 \mathrm{~Hz}$ where it very slightly increases in magnitude. It is likely that the average flow response actually approaches $0 \frac{\mu L}{s}$ but is not reflected in the data due to measurement error.

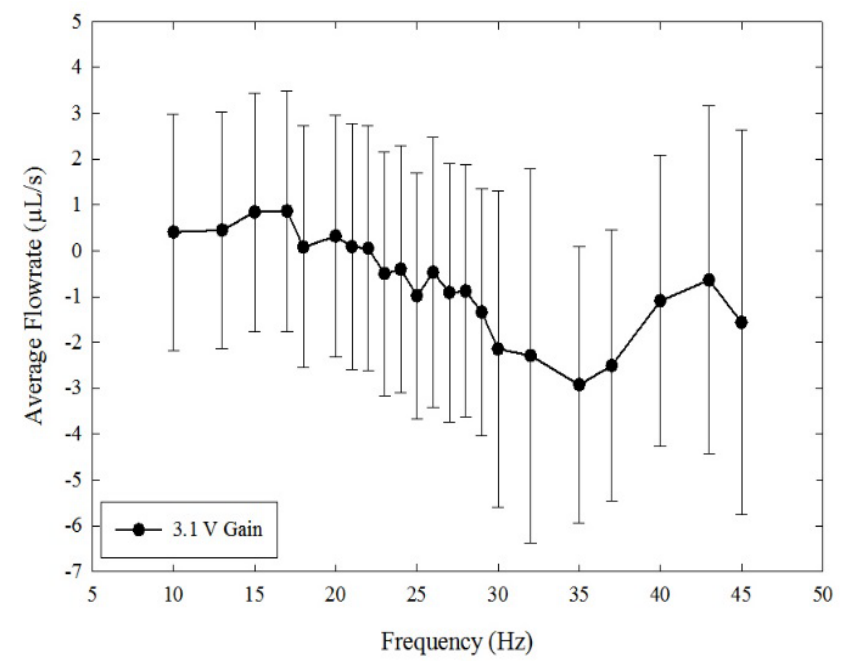

Figure 10. Graph showing the average flow response at several frequencies for a $3.1 \mathrm{~V}$ gain excitation applied at a location 0.625 inch from the end

The average flow response caused by applying perturbations to the test section at a location of 0.875 inch, measured from an end, is shown in Figure 12. Results of this study were extremely similar to those of the study conducted at a location 0.375 inch from the end. A small variation in magnitude of the average flow response over the range of frequencies was observed with a negative peak at $32 \mathrm{~Hz}$. In general, the average flow response has a magnitude of $-1-0.5 \frac{\mu L}{s}$ except at the band of frequencies $25-37 \mathrm{~Hz}$ where it increases in magnitude. The cases where it did not approach nearly $0 \frac{\mu L}{s}$ were attributed to measurement error.

Finally, the average flow response caused by applying perturbations to the test section at a location of 1.125 inches, measured from an end, is shown in Figure 11. A significant variation in magnitude and direction of the average flow response over the range of frequencies is observed with small positive peaks at $27 \mathrm{~Hz}$ and $38 \mathrm{~Hz}$ and two very large negative peaks at $27 \mathrm{~Hz}$ and $33 \mathrm{~Hz}$. The large peaks were observed to occur due to complete pinching closed of the compliant test section over a perturbation cycle at this location which appears to cause the vibrational effects inducing a flow response to be greatly amplified. The occurrence of two peaks also suggests that multiple resonance frequencies are involved in the system and not only that of the compliant test section as expected. For example, it may be that the resonance frequency of the test apparatus also creates a significant flow response. In general, the average flow response has a magnitude of $0 \frac{\mu L}{s}$ except at the band of frequencies $23-37 \mathrm{~Hz}$ where it increases in magnitude.

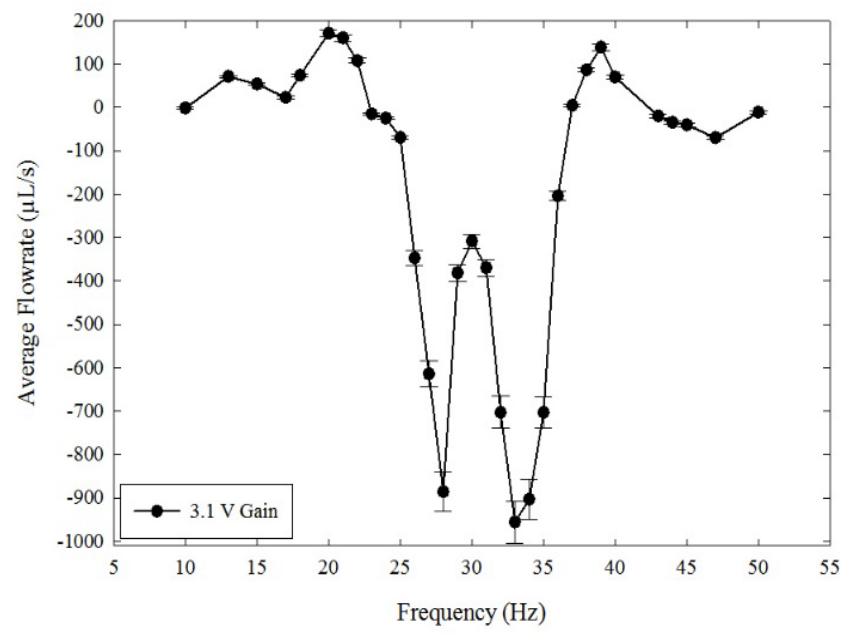

Figure 11. Graph showing the average flow response at several frequencies for a $3.1 \mathrm{~V}$ gain excitation applied at a location 1.125 inches from the end

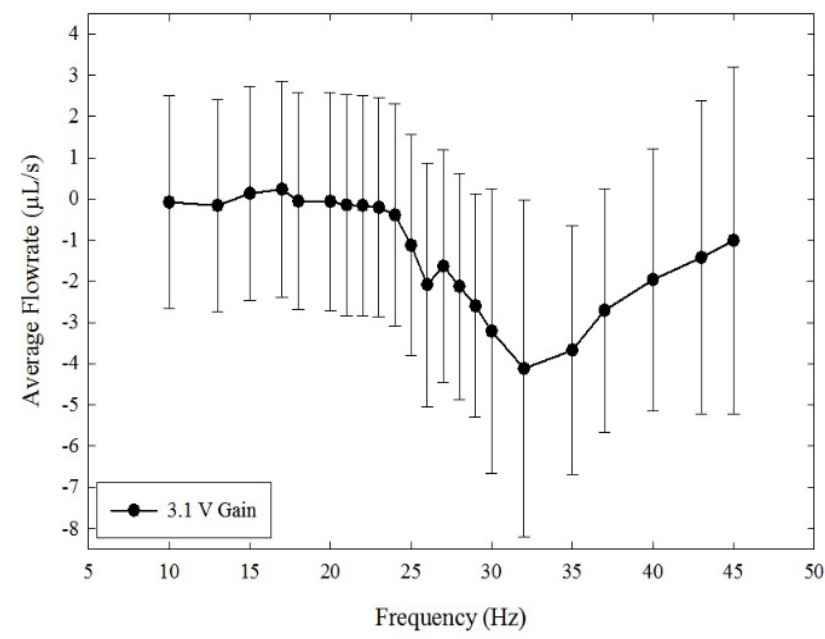

Figure 12. Graph showing the average flow response at several frequencies for a $3.1 \mathrm{~V}$ gain excitation applied at a location 0.875 inch from the end

\subsection{A Fourier Analysis of Pumping}

An FFT analysis of the flow response was conducted at every frequency for a sweep at all five locations to determine the magnitudes of the fluid oscillations and net pumping responses. The FFT obtained at each frequency was gathered into a single graph for an easy comparison of the flow response, Figure 13 (a)-(e). An FFT at a single frequency of $34 \mathrm{~Hz}$ is also presented and demonstrates the three major peaks observed in every FFT response. Results of almost all cases reveal an increase in amplitude from $10 \mathrm{~Hz}$ to between $30-40 \mathrm{~Hz}$ where the response peaks and then starts to decrease. 

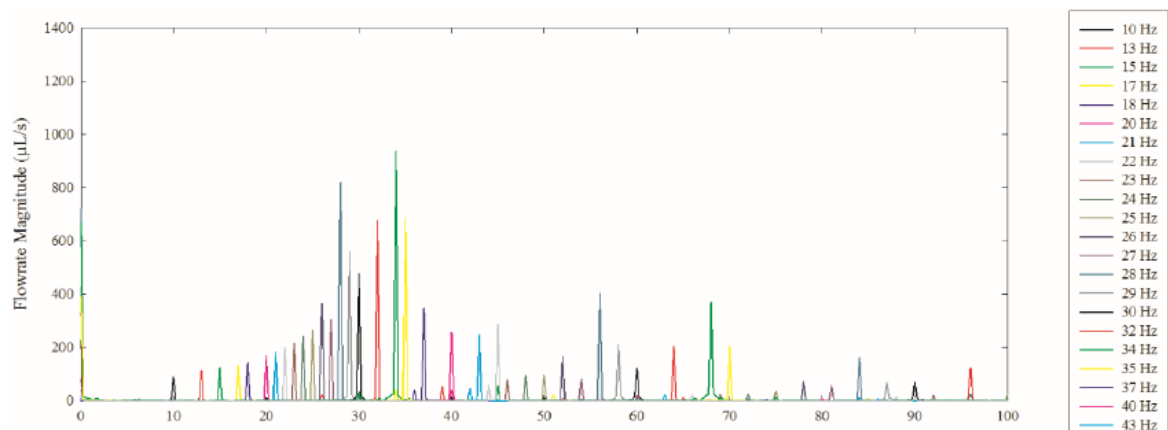

(a) At a location of 0.125 inch
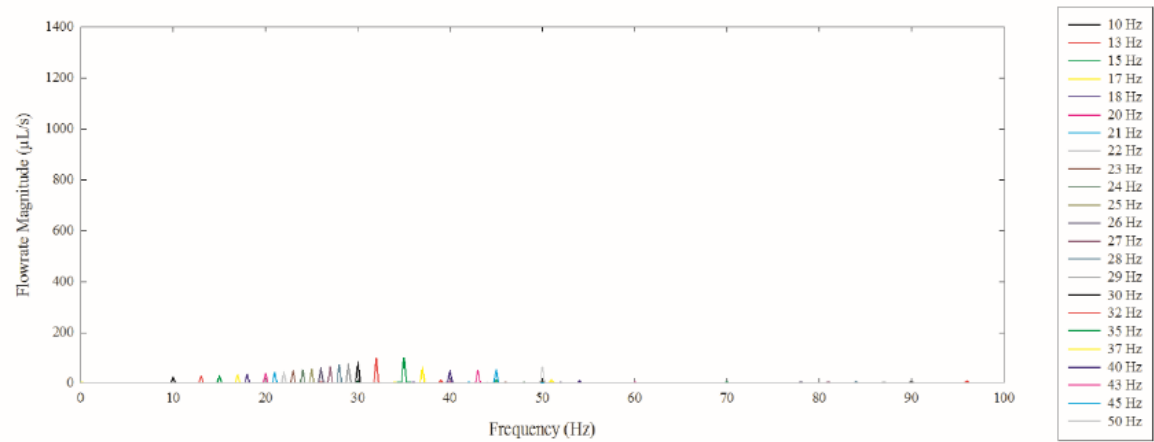

(b) At a location of 0.375 inch.
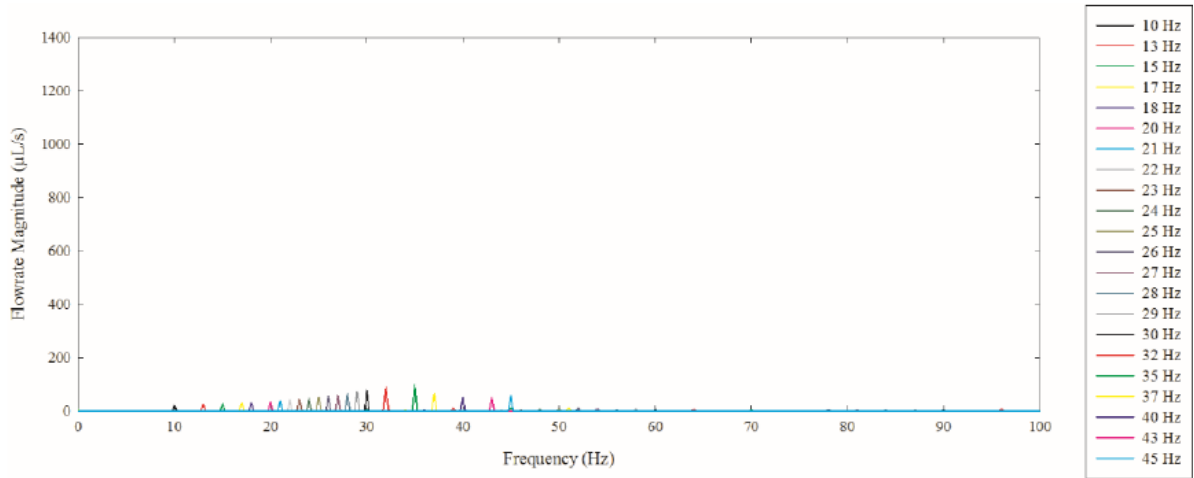

(c) At a location of 0.625 inch.

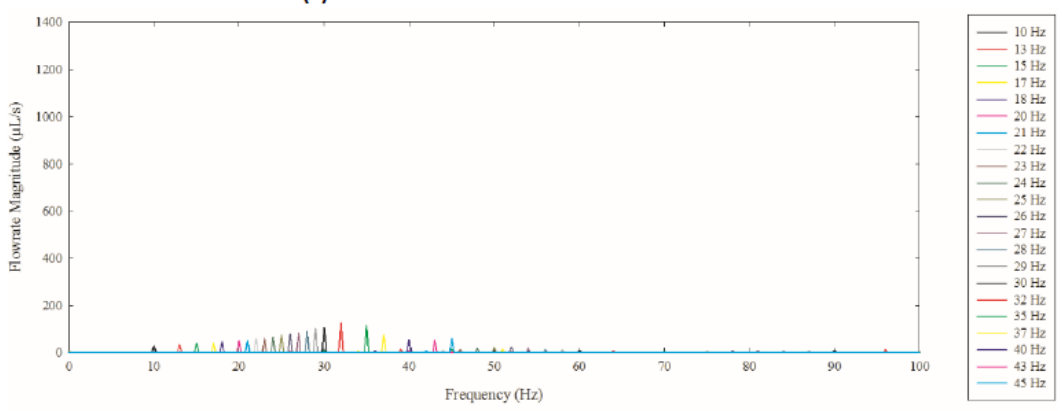

(d) At a location of 0.875 inch.

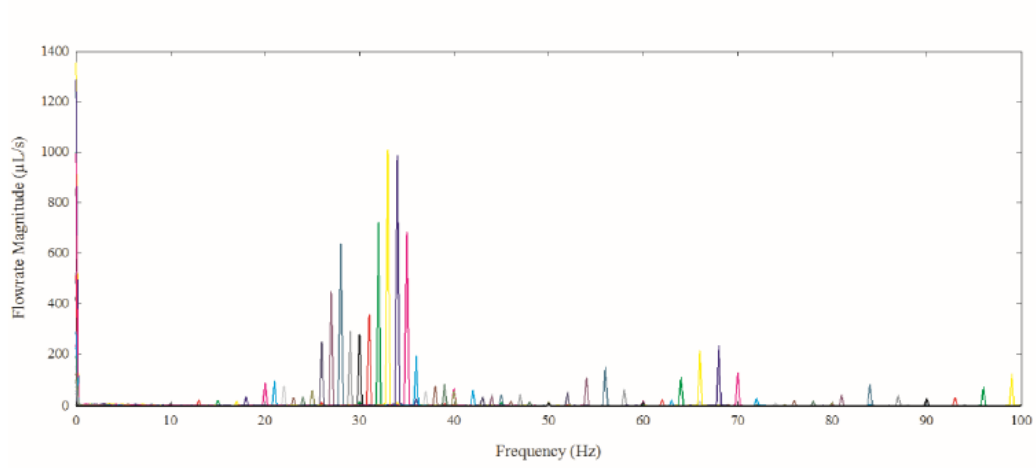

(e) At a location 1.125 inch.

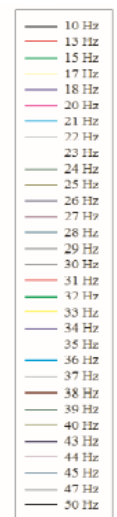

Figure 13. Graph showing a collection of FFT responses for the sweep of frequencies studied experimentally at all five locations (a) through (e) 


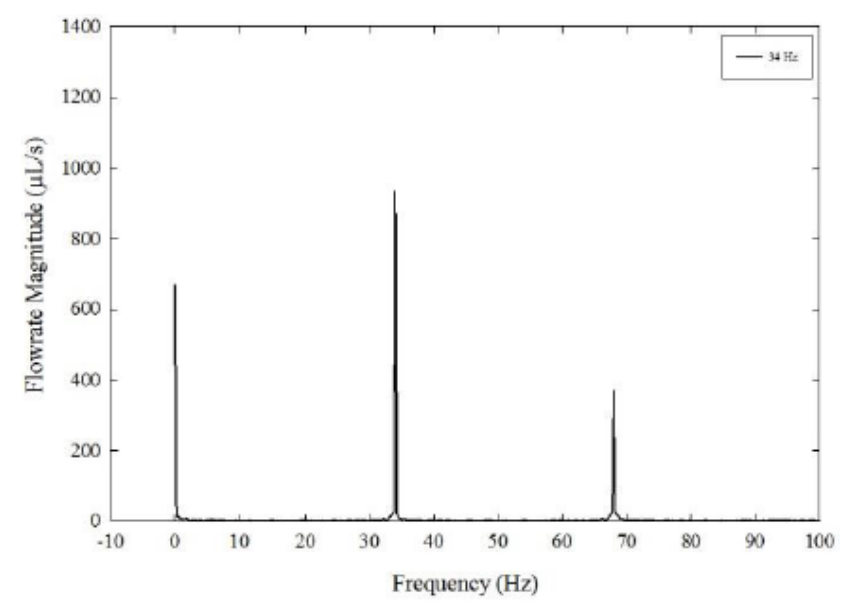

Figure 14. Graph showing a single FFT response at $34 \mathrm{~Hz}$ for a $3.1 \mathrm{~V}$ gain excitation applied at a location of 0.125 inch.

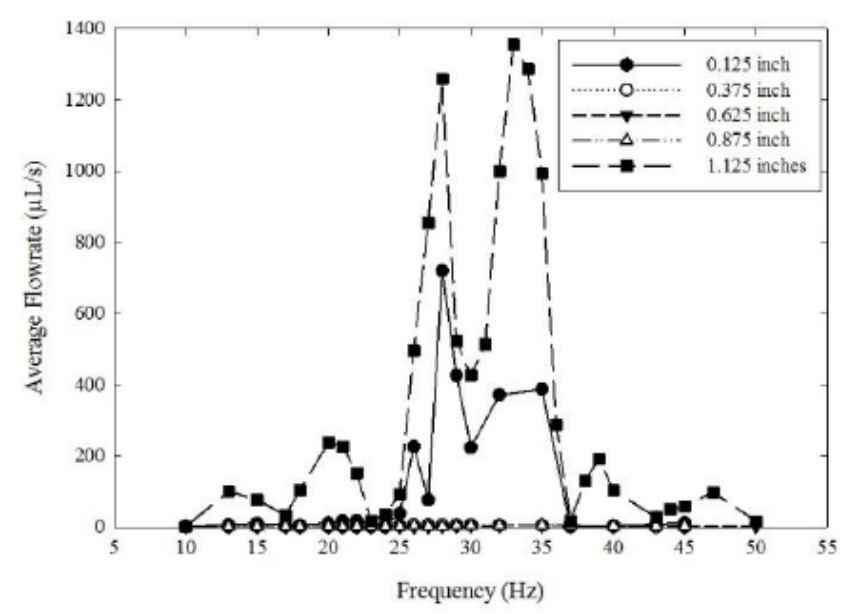

Figure 15. Graph showing the A0 coefficient of the average flow response for a $3.1 \mathrm{~V}$ gain excitation applied at several locations

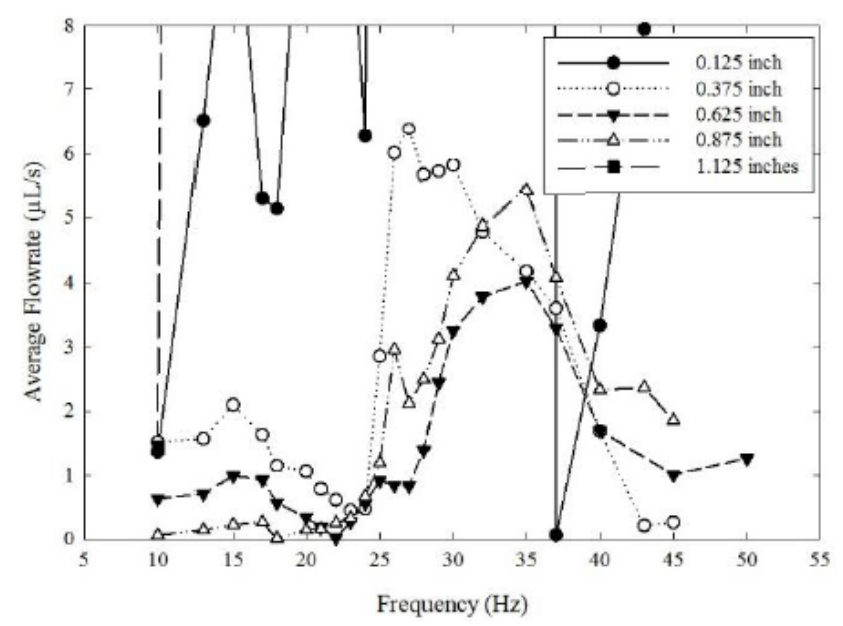

Figure 16. Magnified graph showing the $A_{0}$ coefficient of the average flow response for a $3.1 \mathrm{~V}$ gain excitation applied at several locations

The highest peak for each FFT conducted at some applied frequency always occurred at that frequency, except for a few cases where it occurred at $0 \mathrm{~Hz}$, and exhibited a few smaller peaks at several higher frequencies. Two interesting behaviors are observed in every FFT. The large amplitude occurring at each frequency studied is reflective of the amplitude of sinusoidal oscillations of fluid in response to the applied perturbation. This shows that the fluid oscillates with large displacements but does not produce circulation. Secondly, a non-zero amplitude of various magnitudes occurs at a frequency of $0 \mathrm{~Hz}$ in each $\mathrm{FFT}$, defined by the $\mathrm{A}_{0}$ coefficient, and is reflective of the net pumping of the fluid in response to the applied perturbation. The amplitude of net pumping was much smaller than the oscillatory displacements except at the end locations of the test section where it was nearly equal or greater. A graph of the $A_{0}$ coefficient is presented for each FFT collection as varied by location and illustrates an average flow response similar to those obtained experimentally, Figure 15 and Figure 16.

\section{Conclusions}

The study of periodic perturbations applied to a compliant millitube section of a closed-loop system of tubing demonstrated a variable circulation of internal fluid. The results provide some useful conclusions about the nature of pumping fluid as caused by periodic perturbations of a compliant tube structure. A positive net displacement of fluid was observed to occur in the closedloop system when the compliant test section was perturbed. A graphical presentation of the average flow response with frequency illustrated a significant increase in net fluid displacement at a small band of frequencies from $23-37 \mathrm{~Hz}$. The overall trend is similar to an FFT, and is indicative of a contributing resonance frequency as predicted by vibration theory of elastic structures. A large oscillatory fluid response was also observed to occur in the closed-loop system when the compliant test section was perturbed as predicted by the theory of fluids. It is apparent that the periodic perturbations caused the fluid to be displaced, but only the vibrations were responsible for the net displacement. The magnitude of the flow was determined to be controllable by varying frequency and location of the applied perturbations The flow direction was controllable only at the end locations of 0.125 inch and 1.125 inches by varying the frequency of the applied perturbations. The observed pumping effect was very similar to impedance pumping except that it demonstrated a significant change in magnitude when pinching the compliant millitube section fully closed. Overall, the results obtained in this study verified that perturbations do create a pumping effect that significantly varies with at least excitation frequency and location. Future studies will provide a better understanding of this behavior and progress towards its implementation as a pumping mechanism in biological applications

\section{References}

[1] M. Moser, J. W. Huang, G. S. Schwarz, T. Kenner, A. Noordergraaf, Impedance defined flow. generalisation of william harvey's concept of the circulation-370 years later, International J. of Cardiovascular Med. Sci. 1 (3/4) (1998) 205-211.

[2] T. Kenner, M. Moser, I. Tanev, K. Ono, The liebau-effect or on the optimal use of energy for the circulation of blood, Scripta Medica (BRNO) 73 (2000) 9-14.

[3] W. Wang, S. Soper (Eds.), Bio-MEMS: Technologies and Applications, CRC Press, Bota Raton, Florida, 2006. 
[4] P. Gravesen, J. Branebjerg, O. S. Jensen, Microfluidics-a review, Journal of Micromechanics and Microengineering 3 (4) (1993) 168.

[5] B. D. Iverson, S. V. Garimella, Recent advances in microscale pumping technologies: a review and evaluation, Microfluidics and Nanofluidics 5 (2) (2008) 145-174.

[6] D. J. Laser, J. G. Santiago, A review of micropumps, Journal of Micromechanics and Microengineering 14 (6) (2004) R35-R64.

[7] N. Nguyen, X. Huang, T. Chuan, Mems-micropumps: A review, ASME Journal of Fluids Engineering 124 (2) (2002) 384-392.

[8] G. Lindner, Sensors and actuators based on surface acoustic waves propagating along solid-liquid interfaces, Journal of Physics D: Applied Physics 41 (12) (2008) 123002.

[9] G. Liebau, Aus welchem grunde bleibt die blutförderung durch das herz bei valvulärem versagen erhalten, Verh Dtsch Ges Kreislaufforsch 45 (1956) 481-488.

[10] C. Lee, H. Chang, C. Wen, A mems-based valveless impedance pump utilizing electromagnetic actuation, Journal of Micromechanics and Microengineering 18 (3) (2008) 035044.

[11] D. Rinderknecht, A. I. Hickerson, M. Gharib, A valveless micro impedance pump driven by electromagnetic actuation, Journal of Micromechanics and Microengineering 15 (4) (2005) 861.
[12] C. Y. Wen, C. H. Cheng, C. N. Jian, T. A. Nguyen, C. Y. Hsu, Y. R. Su, A valveless micro impedance pump driven by pzt actuation, Materials Science Forum 505-507 (2005) 127-132.

[13] A. I. Hickerson, D. Rinderknecht, M. Gharib, Experimental study of the behavior of a valveless impedance pump, Experiments in Fluids 38 (4) (2005) 534-540.

[14] J. T. Ottesen, Valveless pumping in a fluid-filled closed elastic tube-system: one-dimensional theory with experimental validation, Journal of Mathematical Biology 46 (4) (2003) 309-332.

[15] C. Koch, V. Remcho, J. Ingle, \{PDMS $\}$ and tubing-based peristaltic micropumps with direct actuation, Sensors and Actuators B: Chemical 135 (2) (2009) 664-670.

[16] V. Shkolnikov, J. Ramunas, J. G. Santiago, A self-priming, rollerfree, miniature, peristaltic pump operable with a single, reciprocating actuator, Sensors and Actuators A: Physical 160 (1-2) (2010) $141-146$

[17] N. J. Graf, M. T. Bowser, A soft-polymer piezoelectric bimorph cantilever-actuated peristaltic micropump, Lab Chip 8 (10) (2008) 1664-1670.

[18] S. G. Kelly, Mechanical Vibrations: Theory and Applications, Cengage Learning, Stamford, CT, 2012. 